\title{
Hipertensão Arterial Experimental e Prenhez em Ratas: Repercussões no Peso da Placenta e no Índice Placentário
}

\author{
Experimental Hypertension and Pregnancy in Rats: \\ Repercussion Regarding Placental Weight and Placental index \\ Rogério Dias ${ }^{1}$, Marilza Vieira Cunha Rudge ${ }^{1}$, José Carlos Souza Trindade ${ }^{2}$
}

RESUM0

Objetivo: estudar as repercussões da hipertensão sobre o peso da placenta e índice placentário. Métodos: foram utilizadas 82 ratas virgens da linhagem Wistar em idade de reprodução. Após a indução da hipertensão arterial experimental (Modelo Goldblatt I - 1 rim-1 clipe) as ratas foram sorteadas para compor os 4 grandes grupos experimentais (controle, manipulação, nefrectomia e hipertensão). A seguir, as ratas foram distribuidas por sorteio em 8 subgrupos, sendo quatro prenhes $(P)$ e quatro não-prenhes. Após acasalamento, dos quatro grupos prenhes obtivemos com o nascimento dos recém-nascidos (RN) os seguintes grupos: $R N-C$, $R N-M, R N-N$ e $R N-H$, respectivamente, controle, manipulação, nefrectomizado e hipertenso. Resultados: quanto ao peso da placenta, o do grupo $R N-C(\bar{x}=0,58 \pm 0,12)$ foi estatisticamente maior que o de todos os demais grupos. Por outro lado, verifica-se que o peso das placentas provenientes do grupo $R N-M(\bar{x}=0,51 \pm 0,05)$ foi maior que o dos grupos $R N-N(\bar{x}=0,45 \pm 0,07)$ e $R N-H(\bar{x}=0,42 \pm 0,04)$, os quais não diferiram entre si. Os indices placentários dos grupos $P-C$ $(M d=0,1085)$ e $P-M(M d=0,1110)$ não diferiram entre si, mas foram menores que os dos grupos $P-N(M d=0,1175)$ e $P-H(M d=0,1211)$, os quais também não diferiram entre si.

Conclusões: a hipertensão e a nefrectomia unilateral determinaram redução do peso das placentas e aumento do indice placentário, evidenciando repercussões no desenvolvimento placentário e fetal.

PALAVRAS-CHAVE: Hipertensão e gravidez. Hipertensão, alterações placentárias. Hipertensão experimental em ratas.

\section{Introdução}

As dificuldades que envolvem o conhecimento da fisiologia placentária e de suas modificações têm impedido a compreensão do verdadeiro mecanismo pelo qual, em determinados quadros hi-

Trabalho realizado no Laboratório de Investigação Experimental do Departamento de Ginecologia e Obstetrícia, Faculdade de Medicina de Botucatu, UNESP

${ }^{1}$ Departamento de Ginecologia e Obstetrícia, Faculdade de Medicina de Botucatu, UNESP

2 Departamento de Urologia, Faculdade de Medicina de Botucatu, UNESP

Correspondência:

Rogério Dias

Departamento de Ginecologia e Obstetrícia

Faculdade de Medicina de Botucatu - UNESP

Caixa Postal 530

18618-970 - Botucatu - SP

Este trabalho foi parcialmente financiado pelo CNPq Processo $n^{\circ} 300388 / 98-9$ pertensivos, podem ocorrer distúrbios danosos ao concepto $^{1,2}$. Há algum tempo, tornou-se bem definido que a desnutrição materna leva a um retardo do crescimento fetal, em contraposição ao conceito, que por muito tempo predominou, de que o feto é um parasita materno.

A placenta que se acreditava ser um filtro, utiliza-se, na realidade, de uma série de mecanismos para a transferência de nutrientes do sangue materno para o feto ${ }^{1,3}$.

A placenta representa importante papel na relação de nutrientes, que são transferidos da circulação materna para a fetal. Tem, por exemplo, a capacidade de relacionar os aminoácidos, visto que as proteínas não atravessam a barreira placentária.

Vários autores já observaram que diversos fatores, tais como hipertensão, desnutrição materna, infecção intra-uterina e fumo, prejudicam o desenvolvimento placentário, determinando pla- 
centas pequenas e com baixo conteúdo de DNA, RNA e proteínas ${ }^{1,2,4-7}$.

A estas constatações, soma-se o fato de a hipertensão materna provocar diminuição do fluxo sangüineo placentário, com redução na transferência de nutrientes específicos como glicose e aminoácidos. Essa condição resulta em baixo peso fetal e placentário ${ }^{1,6,8-10}$.

O estudo da placenta é importante, pois este órgão se interpõe entre as circulações materna e fetal; vem, ainda, elucidar os distúrbios referentes ao desenvolvimento fetal na gestação complicada pela hipertensão.

Entretanto, o papel deste órgão no controle geral do crescimento fetal ainda é pouco conheci$\mathrm{do}^{1,6,11}$.

A partir destas considerações objetivamos, no presente estudo, verificar a repercussão da hipertensão induzida experimental sobre a prenhez em relação ao peso da placenta e o índice placentário.

\section{Material e Métodos}

Foram utilizadas 82 ratas virgens, Wistar, em idade de reprodução, que foram colocadas em gaiolas individuais, com temperatura ambiente entre $22^{\circ}$ e $25^{\circ} \mathrm{C}$, recebendo água e ração normal para animais de laboratório $\left(\right.$ Batavo $^{\circledR}$ ) ad libitum.

Após a indução da hipertensão arterial experimental as ratas foram distribuídas, por sorteio, em quatro grandes grupos experimentais: controle (1), manipulação (2), nefrectomia (3) e hipertensão (4).

A seguir, as ratas foram novamente sorteadas para compor os subgrupos prenhe $(\mathrm{P}) \mathrm{e}$ não-prenhe (NP), constituindo desta forma 8 grupos experimentais: quatro prenhes e quatro nãoprenhes (Tabela 1).

Tabela 1 - Grupos experimentais e respectivas denominações.

\begin{tabular}{lcc}
\hline Grupos & Prenhe & Não-Prenhe \\
\hline Controle (C) & P-C & NP-C \\
Manipulação (M) & P-M & NP-M \\
Nefrectomia (N) & P-N & NP-N \\
Hipertensão (H) & P-H & NP-H \\
\hline
\end{tabular}

No periodo de acasalamento as ratas dos grupos prenhes foram acasaladas e a prenhez diagnosticada pela análise microscópica do esfregaço vaginal. A presença de espermatozóide caracterizou o "dia zero" da prenhez. No período de prenhez, os animais permaneceram em gaiolas individuais.
Com o nascimento dos recém-nascidos provenientes dos respectivos grupos prenhes, obtivemos os seguintes grupos: RN P-1, RN P-2, RN P-3 e RN P-4 respectivamente, controle, manipulação, nefrectomizado e hipertenso.

Utilizamos os seguintes métodos estatisticos: análise de variância paramétrica ${ }^{12}$, análise de variância não-paramétrica e teste de KruskalWallis ${ }^{13}$.

\section{Resultados}

A Figura 1 evidencia as médias $(\overline{\mathrm{x}})$, desviospadrão (DP), coeficientes de variação (CV) e tamanho amostral (n) do peso da placenta dos recémnascidos provindos de ratas sem tratamento cirúrgico - grupo controle ( $\mathrm{RN}-\mathrm{C})$, com manipulação cirúrgica (RN-M), com nefrectomia (RN-N) e com hipertensão $(\mathrm{RN}-\mathrm{H})$. Pela análise estatística $\mathrm{F}_{\text {calc }}=$ $10,27^{*}, \mathrm{~F}_{\text {crit }}=2,86, \alpha=0,05$ observamos que $\mu \mathrm{RnC}$ $>\mu \mathrm{RN}-\mathrm{M}>(\mu \mathrm{RN}-\mathrm{N}=\mu \mathrm{RN}-\mathrm{H})$ ou seja os grupos RN-N e RN-H não diferiram entre si e apresentaram os menores pesos de placenta em relação ao grupo controle RN-C.

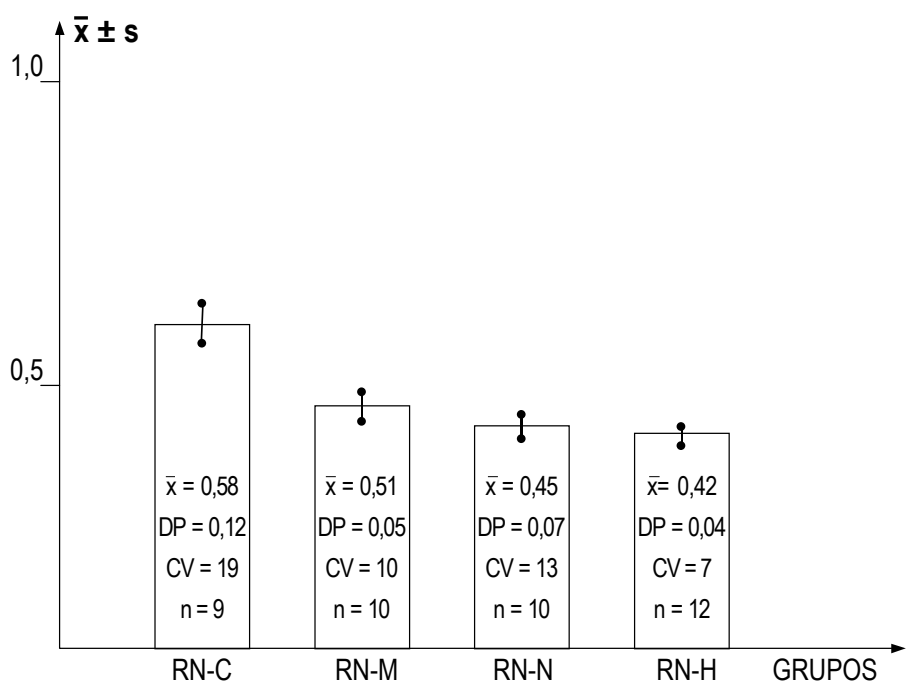

Figura 1 - Valores médios do peso da placenta (em gramas) dos recém-nascidos dos 4 grupos experimentais: (RN-C), (RN-M), (RN-N) E (RN-H). Médias ( $\mathrm{x})$, desvios-padrão (DP), coeficientes de variação (CV) e tamanhos amostrais (n).

Na Tabela 2 observamos a mediana (Md) e tamanho amostral (n) dos valores dos índices placentários em gramas correspondentes aos recém-nascidos e placentas dos 4 grupos experimentais de ratas $\mathrm{P}-\mathrm{C}, \mathrm{P}-\mathrm{M}, \mathrm{P}-\mathrm{N}$ e $\mathrm{P}-\mathrm{H}$. Pela análise estatística calculada $(\mathrm{H})$ observamos que os grupos diferiram da seguinte maneira: $(\mathrm{P}-\mathrm{C}=\mathrm{P}-\mathrm{M})<(\mathrm{P}-\mathrm{N}=\mathrm{P}-\mathrm{H})$, ou seja os maiores índices placentários foram apresentados pelos grupos nefrectomizado e hipertenso. 
Tabela 2 - Valores dos índices placentários (em gramas), correspondentes aos recémnascidos e placentas dos 4 grupos experimentais de ratas: sem tratamento (P-C), com manipulação cirúrgica $(\mathrm{P}-\mathrm{M})$, com nefrectomia $(\mathrm{P}-\mathrm{N})$ e com hipertensão $(\mathrm{P}-\mathrm{H})$. Mediana (Md) e estatística calculada $(\mathrm{H})$, de todos os grupos experimentais.

\begin{tabular}{|c|c|c|c|c|}
\hline Grupos & P-C & P-M & $\mathrm{P}-\mathrm{N}$ & P.H \\
\hline & 0,11 & 0,10 & 0,11 & 0,13 \\
\hline & 0,09 & 0,09 & 0,09 & 0,11 \\
\hline & 0,10 & 0,11 & 0,11 & 0,10 \\
\hline & 0,12 & 0,10 & 0,16 & 0,15 \\
\hline & 0,10 & 0,12 & 0,12 & 0,14 \\
\hline & 0,08 & 0,09 & 0,10 & 0,11 \\
\hline & 0,10 & 0,12 & 0,11 & 0,12 \\
\hline & 0,12 & 0,09 & 0,16 & 0,12 \\
\hline & 0,11 & 0,09 & 0,11 & 0,15 \\
\hline & - & 0,10 & 0,15 & 0,11 \\
\hline & - & - & - & 0,12 \\
\hline & - & - & - & 0,12 \\
\hline$n$ & 9 & 10 & 10 & 12 \\
\hline $\mathrm{Md}$ & 0,10 & 0,11 & 0,11 & 0,12 \\
\hline $\begin{array}{l}\mathrm{N}=41 \\
\mathrm{R}_{1}=137,5 \\
\mathrm{R}_{1}=15,3\end{array}$ & $\begin{array}{l}K=4 \\
R_{2}=139,5 \\
R_{2}=13,9 \\
H=13,4\end{array}$ & $\begin{array}{l}R_{3}=254,5 \\
R_{3}=25,4 \\
p<0,01\end{array}$ & & \\
\hline
\end{tabular}

A Figura 2 evidencia as médias $(\overline{\mathrm{x}})$, desviospadrão (DP), coeficientes de variação $(\mathrm{CV})$ e tamanho amostral (n) do peso corpóreo dos recém-nascidos provindos de ratas sem tratamento cirúrgico - grupo controle (RN-C), com manipulação cirúrgica (RN-M), com nefrectomia $(\mathrm{RN}-\mathrm{N})$ e com hipertensão $(\mathrm{RN}-\mathrm{H})$. Pela análise estatística $\mathrm{F}_{\text {calc }}=$ $37,81^{*}, \mathrm{~F}_{\text {crit }}=2,86, \alpha=0,05$ observamos que ( $\mu \mathrm{RN}$ $\mathrm{C}=\mu \mathrm{RN}-\mathrm{M})>\mu \mathrm{RN}-\mathrm{N}>\mu \mathrm{RN}-\mathrm{H}$, ou seja, os grupos $\mathrm{RN}-\mathrm{N}$ e RN-H apresentaram os menores pesos corpóreos em relação ao grupo controle $\mathrm{RN}-\mathrm{C}$.

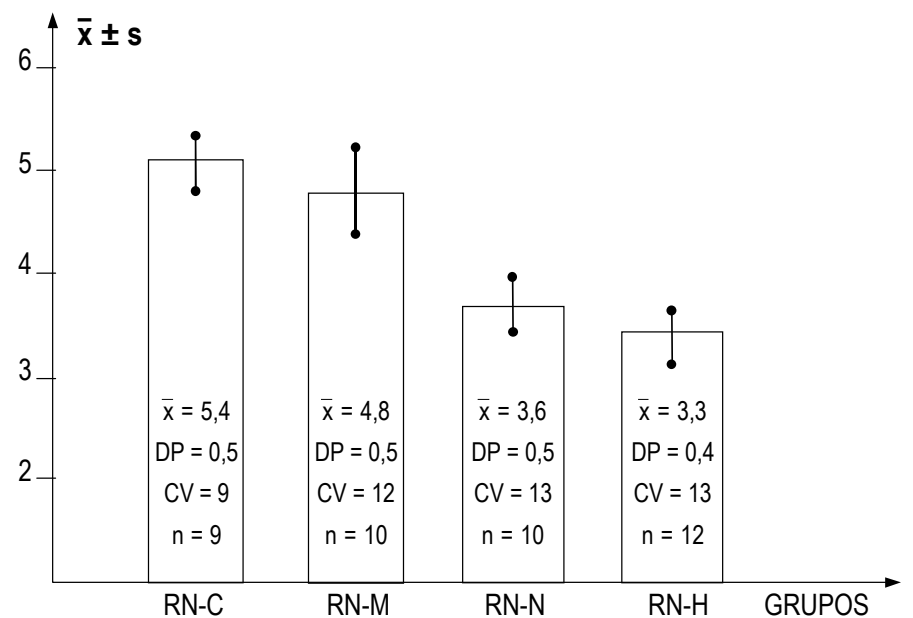

Figura 2 - Valores médios do peso corpóreo (em gramas) dos recém-nascidos dos 4 grupos experimentais: RN-C, RN-M, RN-N e RN-H. Médias (x), desvios-padrão (DP), coeficientes de variação $(\mathrm{CV})$ e tamanhos amostrais (n).

\section{Discussão}

O papel da placenta no controle geral do crescimento fetal ainda é pouco conhecido ${ }^{1,14,15}$.

Este fato constitui uma lacuna importante em nossos conhecimentos, porque provavelmente a placenta, juntamente com o coração do feto, é órgão muito importante durante a vida intrauterina. A placenta desempenha, entre outras, a função de respiração, de excreção, de hematopoese e ainda tem a função de produzir hormônios, todas diretamente relacionadas com a nutrição fetal e destinadas a preparar o feto para a vida extrauterina ${ }^{1,14}$.

Vários autores observaram redução no peso placentário, tanto na espécie humana como em outros animais, devida a diversas causas: insuficiência placentária, infecção intra-uterina, fumo, desnutrição materna, etc. ${ }^{2,6,7,16}$. A idéia de que se poderia avaliar a função placentária através do seu peso foi sugerida por Barcroft ${ }^{17}$. Esse autor referiu que a capacidade da placenta em transferir nutrientes e oxigênio é proporcional a seu tamanho, fator este que explicaria a correlação entre peso placentário e peso fetal.

Atualmente, sabe-se que o grau de transferência de vários elementos difusíveis não é função apenas do tamanho da placenta, embora ainda prevaleça a importância desse parâmetro para avaliação do crescimento fetal ${ }^{2,6}$.

No presente estudo observa-se que o peso da placenta do grupo controle RN P-1 é estatisticamente maior que o de todos os demais grupos. Por outro lado, verifica-se que a média dos pesos das placentas provenientes do grupo manipulado foi maior que a dos grupos nefrectomizado e hipertenso, as quais não diferem estatisticamente entre si (Tabela 1 e Figura 1).

Entretanto, deve-se ressaltar que a repercussão foi maior nas placentas dos grupos nefrectomizados e hipertensos. Essas observações evidenciam que discretas alterações metabólicas ou de outra natureza são suficientes para determinar repercussões no peso da placenta, que indiretamente podem refletir alteração de fisiologia placentária.

Vários autores observaram que a hipertensão leva à redução do fluxo sangüíneo placentário e a desnutrição fetal e, consequentemente, ao baixo peso fetal e placentário ${ }^{6,18,19}$. Entretanto, Karlsson et al. ${ }^{8}$, em estudo experimental com ratas grávidas tornadas hipertensas pela técnica de Goldblatt I, observaram redução de fluxo sangüíneo miometrial e placentário nesses animais, porém, não constataram diminuição do peso placentário.

Nossos dados estão em desacordo com os de Karlsson et al. ${ }^{8}$, porém concordes com os de Nitzan 
et al. ${ }^{4}$ que, estudando o efeito da hipertensão induzida por nefrectomia subtotal em ratas prenhes, observaram que o peso placentário das ratas hipertensas era menor quando comparado com o grupo controle normal. Wigglesworth ${ }^{10}$, produzindo insuficiência vascular causada por ligadura de artéria uterina em um dos cornos do útero de rata, e sendo o outro utilizado como controle, observou correlação entre retardo de crescimento placentário e o de outros orgãos fetais.

Cibils ${ }^{14}$ observou, em humanos, que as placentas de mães hipertensas eram significativamente menores em tamanho e peso quando comparadas as de mães normais, sugerindo que o processo patológico interfere no crescimento normal da placenta.

As placentas humanas de recém-nascidos com baixo peso para a idade gestacional apresentaram alta incidência de alterações circulatórias, entre as quais o enfarte e essas alterações seriam responsáveis pelo baixo peso do recém-nascido $^{1,20}$. Entretanto, outros autores admitem que a placenta apresenta grande reserva funcional e apenas enfartes superiores a $10 \%$ da área placentária determinariam redução no suprimento fetal e, conseqüentemente desnutrição fetal e baixo peso placentário ${ }^{1,21}$.

Tem sido demonstrada que, ao nascimento, há uma correlação positiva entre o peso placentário e peso fetal, mas a base fisiológica desta correlação ainda está obscura ${ }^{22}$. Até o momento não se tem um método para avaliar o crescimento intrauterino da placenta no evoluir da gestação ${ }^{1,6,23}$. Entretanto, alguns autores relatam que o crescimento placentário pode ser acompanhado por meio de parâmetros como DNA, RNA e proteínas e da enzima ribonuclease (RNase) presente no núcleo e citoplasma das células ${ }^{2,7}$.

Entretanto, o índice placentário (relação peso placenta/peso do feto) é usado com padrão de função placentária ${ }^{1,23,24,25}$.

Os valores dos índices placentários dos 4 grupos experimentais em estudo mostram que os grupos controle e manipulação não diferem estatisticamente entre si. Por outro lado, esses dois grupos apresentam índices placentários estatisticamente menores do que os grupos nefrectomizado e hipertensão, os quais também não diferem estatisticamente entre si.

Verifica-se estes últimos grupos apresentaram os maiores índices placentários, o que traduz uma maior repercussão da nefrectomia e da hipertensão no desenvolvimento placentário fetal. Entretanto, se confrontarmos os gráficos dos pesos placentários e dos pesos fetais observamos que foi o peso fetal o fator determinante do maior índice placentário, tanto no grupo nefrectomizado com no grupo hipertenso.
Nas condições de nosso trabalho e com base nos resultados obtidos, podemos concluir que a hipertensão e a nefrectomia unilateral determinaram redução do peso das placentas e aumento do índice placentário, evidenciando repercussões no desenvolvimento placentário e fetal.

\section{SUMMARY}

Purpose: to study the repercussion regarding placental weight and placental index determined by induced experimental hypertension in rats.

Methods: a total of 82 rats in reproductive age were used. They were randomly assigned to 4 different groups (control, handled, nephrectomy and hypertension) and renal hypertension was produced by a controlled constriction of the main left renal artery, according to the technique described by Goldblatt, and contralateral nephrectomy (Goldblatt I one kidney-one clip model). Furthermore, they were distributed into non-pregnant groups and pregnant $(P)$ groups. From the pregnant groups, the following newborn groups were obtained: $R N-C$ (control - newborn group from the pregnant rats without surgical treatment), $R N-M$ (manipulation - newborn group from the pregnant rats with surgical manipulation), $R N-N$ (nephrectomy newborn group from the pregnant rats with nephrectomy) and $R N-H$ (hypertension - newborn group from pregnant rats with hypertension).

Results: the $R N-C$ newborn group $(\bar{x}=0.58 \pm 0,12)$ showed placental weight higher than the other three groups ( $R N-M$ : $\bar{x}=0.51 \pm 0.05 ; R N-N: \bar{x}=0.45 \pm 0.07$ and $R N-H: \bar{x}=0.42 \pm$ $0.04)$. On the other hand, it was possible to observe that the placental weight of the $R N-M$ was higher than that of $R N-N$ and $R N-H$, respectively, but no difference was observed between the $R N-N$ and $R N-H$ groups. The placental index showed no difference between $P-C(M d=0.1085)$ and $P-M$ $(M d=0.1110)$, and also between $P-N(0.1175)$ and $P-H$ (0.1211), but it was observed that the placental indexes of $P$ $C$ and $P-M$ were smaller that those of $P-N$ and $P-H$.

Conclusion: unilateral nephrectomy and hypertension determined a reduction in placental weight and an increase in the placental index, showing a repercussion regarding placental and fetal development.

KEY WORDS: Hypertension and pregnancy. Hypertension, placental disorders. Experimental hypertension in rats.

\section{Referências}

1. Kingdom JC, Kaufmann P. Oxygen and placental vascular development. Adv Exp Med Biol 1999; 474:259-75. 
2. Nóbrega FJ, Tonete SSQ, Sartor MEA, Cury PR. Estudo experimental do crescimento placentário na desnutrição protéico-calórica. J Pediatr 1979; 46:82-92.

3. Rudge MVC, Trindade CEP. A placenta na desnutrição protéico-calórica. In: Nóbrega FJ, editor. Desnutrição Intra-uterina e Pós Natal. $1^{\text {a }}$ ed. São Paulo: Panamed; 1981. p.144-8.

4. Nitzan M, Orloff S, Chrzanowska BL, Schulman JD. Intrauterine growth retardation in renal insufficiency: An experimental model in the rat. Am J Obstet Gynecol 1979; 133:40-3.

5. Rosso P. Maternal-fetal exchange during protein malnutrition in the rat. Placental transfer of glucose and a nonmetabolizable glucose analog. J Nutr 1977; 107:2006-10.

6. Rudge MVC, Gomes CMM, Calderon IMP, et al. Study of the evolution of the placenta and fetal pancreas in the pathophysiology of intrauterine growth retardation due to restricted maternal diet. São Paulo Med J 1999; 117:49-56.

7. Trindade CEP, Nóbrega FJ, Rudge MVC, et al. Relação do peso de recém-nascidos e placentas. Estudo em recém-nascidos de termo, pré-termo e póstermo, de pesos adequado, baixo e grande para a idade gestacional. J Pediatr 1979; 46:208-14.

8. Karlsson K, Ljungblad U, Lundgren Y. Blood flow of the reproductive system in renal hypertensive rats during pregnancy. Am J Obstet Gynecol 1982; 142:1039-44.

9. Khoury A, Wasserstrum N, Rudelstorfer R, Nuwayhid B, Khakmahd K, Brinkman CR $3^{\text {rd }}$. Experimental hypertension in pregnant sheep. III. Central hemodynamic alterations in the one-kidney model. Am J Obstet Gynecol 1986; 155:1231-6.

10.Wigglesworth JS. Experimental growth retardation in foetal rat. J Pathol Bacteriol 1964; 88:1-13.

11.Di Matteo MS, Sala MM. Desenvolvimento e estrutura da placenta. In: Garcia A, Azouber R, editores. A Placenta Humana: morfologia e patologia fetal e perinatal. $1^{\mathrm{a}}$ ed. Rio de Janeiro: Atheneu; 1986. p.1-14.

12.Snedecor GW, Cochran WG. Statistical methods. $7^{\text {th }}$ ed. Ames: The Iowa State University Press;
1980. p.505.

13. Siegel S. Estatística não paramétrica. $1^{a}$ ed. São Paulo: McGraw Hill do Brasil; 1975. p.350.

14. Cibils LA. The placenta and newborn infant in hypertensive conditions. Am J Obstet Gynecol 1974; 118:256-70.

15.Rosso P. Changes in the transfer of nutrients across the placenta during normal gestation in the rat. Am J Obstet Gynecol 1975; 122:761-6.

16.Winick M. Cellular changes during placental and fetal growth. Am J Obstet Gynecol 1971; 109:166-76.

17.Barcroft J. Researches on prenatal life. $1^{\text {st }}$ ed. Springfield: Charles C. Thomas; 1947. p.42-66.

18.Brosens I, Dixon HG, Robertson WB. Fetal growth retardation and the arteries of the placental bed. Br J Obstet Gynaecol 1977; 84:656-63.

19.Wallace JM, Bourke DA, Aitken PP. Nutrition and fetal growth: paradoxical effects in the overnourished adolescent sheep. J Reprod Fertil Suppl 1999; 54:385-99.

20.Scott JM, Jordan JM. Placental insufficiency and small for dates baby. Am J Obstet Gynecol 1972; 113:823-32.

21.Longo LD. Disorders of placental transfer. In: Assali NS, editor Pathophysiology of gestation. $1^{\text {st }}$ ed. New York: Academic Press; 1972. p.40.

22.Zamenhof S, Holzman GB. Study of correlations between neonatal head circumferences, placental parameters and neonatal body weights. Obstet Gynecol 1973; 41:855-9.

23. Sala MM. Estudo do crescimento intra-uterino na segunda metade da gestação. Determinação dos percentis $10^{\circ}, 25^{\circ}, 50^{\circ}, 75^{\circ}$ e $90^{\circ}$, do peso placentário, índice placentário, peso e estatura fetal [tese]. Ribeirão Preto: Universidade de São Paulo; 1977.

24.Little WA. The significance of placental/fetal weight ratios. Am J Obstet Gynecol 1960; 79:134-7.

25.Nummi S. Relative weight of the placenta and perinatal mortality. A retrospective clinical and statistical analysis. Acta Obstet Gynecol Scand Suppl 1972; 17:1-69.

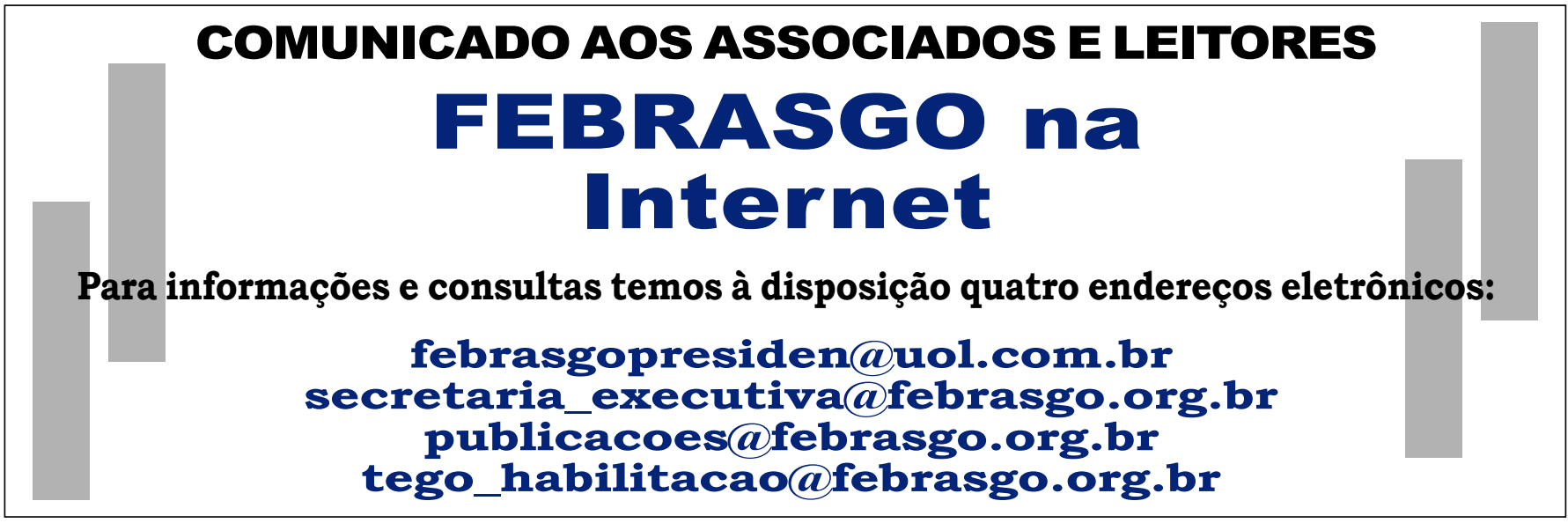

